\title{
SOBRE LOS PRINCIPIOS ÉTICOS EN CONSEJO PSICOLÓGICO Y PSICOTERAPIA
}

\author{
Mark H. Bickhard, \\ Lehigh University
}

\begin{abstract}
RESUMEN
Las cuestiones y principios éticos son de fundamental importancia para las relaciones interpersonales que se dan en el contexto de la psicoterapia. Afirmamos, de hecho, que la relación terapéutica no es solamente una relación a la que se aplican consideraciones éticas, sino que también, y sobre todo,es una relación que, en sl misma, se constituye éticamente. Sin embargo, los fundamentos conceptuales dominantes en la actualidad para la comprensión de la ética y la creación de políticas coherentes son confusos e inadecuados. En particular, la noción de relaciones duales como algo que supone aspectos antiéticos, es conceptualmente vacia. A lo largo de este trabajo argumentaré que esta vacuidad en el concepto no es meramente un error neutro en lo que al pensamiento ético se refiere, sino que es manifiestamente dañino y conlleva consecuencias, ellas sl, antiéticas. El hecho es que siendo conceptualmente vacia, la noción de relación dual es fácilmente invocada para ponerla al servicio de valores intrinsecamente antiéticos o al menos, no revisados con anterioridad. A cambio, yo sugiero una explicación alternativa aplicada particularmente a las violaciones éticas involucradas en las relaciones sexuales entre terapeuta y paciente.
\end{abstract}

PALABRAS ClAVE: Ética, principios éticos, psicoterapia, consejo psicológico, relaciones duales, paternalismo, relaciones sexuales, relación terapeuta-cliente, relaciones sexuales terapeuta-cliente.

\section{ABSTRACT}

Issues of ethics and of principles of ethics are of fundamental importance for the relationships involved in counselling and psychotherapy. The author argues that the therapy relationship is not only a relationship to which ethical considerations apply, but that it is, more deeply, a relationship that is ethically constituted. Nevertheless, the dominant contemporary conceptual foundations for understanding these issues, and for creating policy, are muddled and inadequate. in particular, the notion of a dual relationship as somehow intrinsically involving unethical aspects is conceptually empty. In this paper the author argues that this emptiness is itself not merely a neutral error in ethical thought, but that it is expressly harmful and yields unethical consequences. In particular, in being conceptually empty, the dual relationship notion is easily invoked in the service of intrinsically unethical background values or unexamined values. The author offers an alternative explanation about the ethical violations involved in therapist-client sexual relationships.

KEY WORDS: Ethics, ethical principles, psychotherapy, counseling, dual relationships, patronization, sexual relationships, therapist-client relationships, therapist-client sexual relationships 


\section{RELACIONES DUALES}

Una relación dual es aquella que, sostenida entre dos personas, supone dos o más roles relacionales. Más concretamente, el término suele aplicarse cuando hay poder diferencial entre las dos personas y según los múltiples roles que asuman -con una persona asumiendo uno o varios roles que suponen una gran diferencia de poder en comparación con la otra persona involucrada. Una relación dual en este sentido es tomada como intrínsecamente antiética, generalmente por la potencial explotación a la que la persona con el rol de mayor poder podría someter a la otra.

Un caso paradigmático de esta explotación del diferencial de poder existente en una relación de terapia, es el de las relaciones sexuales que pueden darse entre terapeuta $y$ cliente. Esta explotación es universalmente condenada y la aplicación de la noción de relación dual en esta situación -una relación terapéutica sostenida simultáneamente con relaciones sexuales entre los involucrados es una clara relación dual con un diferencial de poder-. Aquí, el principio de relación dual juzga como antiético algo que de hecho es universalmente considerado como tal.

Sin embargo, este es un razonamiento falaz y peligroso. Constituye una instancia de la clásica falacia que se basa en el siguiente razonamiento: "Si $A$ entonces $B$ "; si acordamos $B$, entonces concluímos $A$. Una versión simple de esto mismo sería: "Si Johnson mató a Jones (A), entonces Jones está muerto(B)"; si por otra parte decimos que "Jones está muerto(B)", entonces, concluimos que "Johnson mató a Jones(A)". La falacia, ilustrada de esta manera, es aún más clara. En el caso de la noción de relación dual, tenemos una versión implícita de esta misma falacia. Comenzamos con algo como "Si la noción de relación dual es un razonamiento válido para explicar una relación intrínsecamente antiética, entonces, como la existencia de una relación sexual entre terapeuta y cliente es una relación dual, la relación sexual entre ellos es antiética"; estamos de acuerdo en que una relación sexual entre terapeuta y paciente es antiética, por tanto, concluímos que la noción de relación dual explica intrínsecamente una forma antiética de relación. Este estilo de razonamiento, sin embargo, apoya cualquier principio aceptado que tenga como implicación la conclusión de que la confluencia de terapia y relaciones sexuales es antiética. Por ejemplo, podemos extender este razonamiento para el principio de que "cualquier relación en la que las partes bajo consideración están respirando es antiética". Esto ciertamente conllevaria la conclusión deseable que la terapia y las relaciones sexuales son antiéticas, lo que sin embargo no adoptamos como principio. ¿Por qué? Porque es absurdo: toda relación implica necesariamente que haya respiración; este principio es cumplido por todas las relaciones, por tanto el principio no distingue lo ético de 10 antiético.

¿Por qué, sin embargo, nos vemos tan tentados a aceptar esta forma de razonamiento en el caso de las relaciones duales? La respuesta, por supuesto, puede diferir entre aquellos que lo aceptan, pero la mayor fuente para dicha tentación parece ser la intuición de que el diferencial de poder dentro de las relaciones duales intrinsecamente supone riesgos. En particular, está el riesgo de que la explotación potencial a la que puede someterse a quien tiene menos poder será extremadamente tentadora para el más fuerte; o que tal explotación se dé inadvertidamente; o que la explotación sea percibida por parte del miembro menos poderoso, asi sea incorrectamente; o que el potencial de explotación será per se preocupante o confuso. Por lo tanto, parece haber poca duda en cuanto a que éstos son riesgos potenciales de las relaciones duales.

La existencia de riesgos, sin embargo, no significa inevitablemente que algo es antiético: tampoco sirve para diferenciar lo ético de 10 antiético. Sin embargo, el someter a alguien a un riesgo innecesario o sin su consentimiento, sí puede ser considerado como algo antiético. Luego, quizás, es más bien esto último lo que determina que una relación dual sea antiética.

Como las relaciones duales involucran sus propias formas particulares de riesgos, entonces someter innecesariamente a alguien a dichos riesgos o sin su consentimiento puede ser señalado como antiético. El criterio "sin su consentimiento" no es el más adecuado para abogar por el principio de relación dual. Ciertamente, puede aceptarse que hacerle algo a 
alguien, o con alguien, o por alguien sin o en contra de su consentimiento es, claramente, antiético o muy peligroso éticamente. Pero to que ocurre es que basarse en cuestiones de consentimiento para hacer juicios éticos implica que el aspecto antiético de las relaciones duales sea la violación del consentimiento y no la relación dual per se. La noción de relación dual entonces será éticamente irrelevante.

La cuestión ética de la explotación per se no puede apoyar el principio de relación dual. Se aceptará que la explotación es algo antiético, pero con este principio solo una relación dual en la que se dé explotación será antiética en virtud de tal explotación, no debido a la dualidad. Y las relaciones duales en las que tal explotación no ocurra no serán intrínsecamente antiéticas. De nuevo, la noción de relación dual per se será éticamente irrelevante.

De manera similar, el criterio de exponer a alguien a un "riesgo innecesario" es inadecuado para explicar un carácter intrínsecamente antiético de las relaciones duales. La razón aquí es, simplemente, que para tener fuerza ética, el criterio de "riesgo innecesario" requiere que tal riesgo sea razonablemente evitable -que de hecho sea "innecesario". $Y$ es aquí donde encontramos la mayor vacuidad de la noción de relación dual.

Las relaciones duales suponen sus propios riesgos particulares. Por tanto, mientras una relación dual sea evitable, el involucrar a alguien en ella supondrá que se le está exponiendo a riesgos innecesarios. Desafortunadamente las relaciones duales, y con ellas los riesgos particulares que suponen, no son evitables. La dualidad y, más especificamente, la dualidad en cuanto al diferencial de poder de los roles involucrados, es inevitable en cualquier relación posible. La dualidad es vacia, como lo es el hecho de respirar, como criterio para diferenciar lo ético de lo antiético.

Intercambiar saludos con la persona en la registradora de un supermercado constituye una relación dual. Incluso sin intercambiar el saludo, los roles duales de la transacción financiera y el de respetar el espacio del otro existen. Estos ejemplos son triviales, pero sus implicaciones no lo son: si la noción de relación dual se aplica en instancias de interacción tan triviales, entonces es claro que no puede emplearse para diferenciar entre ético y antiético sin importar qué tanto se aplica también en instancias paradigmáticas como la de terapia y las relaciones sexuales. Las relaciones duales, al ser inevitables en general, también son inevitables en muchas otras circunstancias menos triviales. En el caso de una relación profesional, consideremos el caso de un terapeuta que vive en un pueblo o ciudad pequeña: muchos de sus clientes inevitablemente serán personas que él o ella conozca.

La aplicación de la noción de relación dual como criterio de ética en tal caso no es algo meramente trivial, sino potencialmente muy dañino. La negación de los servicios en tales comunidades, la preocupación del terapeuta por la aplicabilidad de tales "principios éticos" en su práctica, y la confusión por falta de una guia ética por parte de un principio tan vacio. son unos de los peligros posibles. Qué significado puede tener una base moral que solamente es aplicable en una gran ciudad y en función del anonimato de una comunidad, como por ejemplo, la ciudad de Manhattan?

En un ambiente educativo, en situaciones de supervisión, cursos experienciales, cursos tutoriales y evaluaciones profesionales hechas a los estudiantes, habrá combinaciones de roles de profesor-terapeuta y de profesor-evaluador, y en todas ellas habrá relaciones duales con diferencias de poder. Todas poseen intrínsecamente los riesgos particulares de las relaciones duales -todos los riesgos concemientes a posible explotación, miedos de explotación y demás, propios de la diferencia de poder dada en tales relaciones. De nuevo, esto no lleva a la condenación ética de tales roles -aunque puede alertar en cuanto a algunos riesgos posibles. En cambio, esto proporciona aún más ejemplos de la absoluta vacuidad de la relación dual como criterio ético.

La abolición de "la relación dual" como criterio ético es muy importante, pero es sólo una parte de la tarea. Es únicamente la parte conceptual de la tarea. Queda pendiente la parte ética de deshacer y prevenir el gran daño que se ha hecho, y que puede ser hecho, como consecuencia de la popularidad de un principio tan vacio dentro de la ética. El daño es, en gran parte, resultado del hecho de que al aplicar como principio ético algo que es conceptualmente vacio y que es aplicado a 
todo, todos los criterios reales para diferenciar aquello que se condena y aquello que no se condena son, inevitablemente, intuitivos, escondidos tras el alegado principio y por tanto, no abiertos a una discusion racional. Las razones reales, por ejemplo, por las que la terapia $y$ las relaciones sexuales son condenadas 0 deben serlo, y por las que las relaciones profesor-evaluador, no lo son y no deben serto, no están disponibles para consideración ni discusión. Esto es, el daño patrocinado por el principio de relación dual no puede ser entendido sin reconocer primero que es solamente un principio particular y no un valor. La noción de que las relaciones duales son intrínsecamente antiéticas es un principio conceptual puesto al servicio de ciertos valores. Es un intento de imposición de dichos valores, inventado precisamente con tal propósito; no tiene una fuerza moral por si misma, pero parece que la adquiriera sólo por estar al servicio de tales valores, en virtud de su aplicabilidad como un sustituto de esos otros valores. En este caso, los valores relevantes históricamente son aquellos concernientes a la ética de la relación terapéutica. La noción de relación dual supuestamente proporciona un principio por el cual las relaciones antiéticas pueden ser definidas, distinguidas y sancionadas, y que sustituye a valores éticos subyacentes menos claros. Notemos que esta función de la noción es, de hecho, la única manera posible de introducirla, ya que ella no diferencia -estrictamente- ninguna relación de otra. Sin los valores subyacentes en cuyo servicio el principio de relación dual supuestamente es aplicado, no habria forma de diferenciar la relación con la empleada del supermercado de las relaciones sexuales en una situación de terapia.

Lo contrario de esto, sin embargo, es que al ser vacía la noción de relación dual, puede ser aplicada al servicio de cualquier conjunto de valores. Aún más, su función de máscara como valor ético en sí misma opaca tanto la existencia como el contenido de los valores reales subyacentes a ella. Oscurece los valores al servicio de los cuales es aplicada -quizás incluso los oscurece para quienes la están aplicando!.

Algo no gusta y parece malo y -muy seguramente- viola el principio de relación dual, por tanto es antiético y debe ser prohibido y sancionado en caso de violación. Esto, de por si, ya es una situación extremadamente peligrosa. Incluso en este nivel preliminar de análisis, encontramos que una consideración racional por parte del individuo y una discusión racional en la comunidad son inhibidas simplemente porque las cuestiones reales están escondidas.

Cuando cuestiones genuinas son esquivadas en favor de principios vacios, se abre una amplia puerta para cometer abusos -tanto de manera intencional como no intencional. Inclusive para una persona bien intencionada es dificil escapar al efecto dañino del principio vacio de las relaciones duales sin que, de alguna manera, ignore y evada las aseveraciones hechas a favor del mismo principio. Una vez que se le da algún crédito a dicho principio, es imposible pensar claramente o permitirle a alguien más hacerlo, en cuestiones concernientes a las relaciones -después de todo, el principio siempre aplica y, por tanto, siempre habrá que darle crédito. El abuso entonces, intencional o no, se da cuando se usa el principio al servicio de valores que -si son examinados clara y justamente- serán, en el mejor de los casos, debatibles y en el peor, totalmente antiéticos. Tal examen y discusión abierta sin embargo, es precisamente to que se ha excluido al aceptar una premisa de pensamiento vacia.

\section{PATERNALISMO}

Uno de los valores más perniciosos en este campo, al servicio del cual se invoca con frecuencia el principio de relación dual, es aquel de cuidar a otros "por su propio bien". Este es un valor positivo prima fácil en cuanto hace alusión a la vigilancia hecha en favor del bienestar de los otros. Desafortunadamente, con frecuencia es una preocupación por el bienestar de otros en donde tal bienestar es definido, supervisado e impuesto por una persona o personas externas que asumen tal rol sin importar el conocimiento o consentimiento que aquellos protegidos tengan sobre el hecho de que se les está cuidando. Esto, usualmente, constituye una práctica de paternalismo.

El paternalismo está entre los valores más peligrosos que constituyen vicios y excesos 
morales. Es algo muy dificil de combatir, pues se basa en -asi lo proclama- una rectitud moral que se hace "por el propio bien" de alguien. Es algo que es deshumanizante y minimizante. Que se plantea desde una asunción de superioridad de un alguien que infantiliza a otro. Viola intrínsecamente los dere-chos de los adultos y perpetua tal violación, ya que las victimas dejan de ser consideradas como seres humanos adultos, para pasar a ser protegidos por unos benefactores más sabios y conocedores que los primeros.

En ciertas instancias, este paternalismo es apoyado por modelos de personalidad y psicoterapia en donde los individuos, principalmente los que son clientes, son concebidos como intrinsecamente regresivos e infantiles -al menos con relación a su terapeuta-, y condenados a permanecer asi para siempre. El tratamiento dado a clientes o estudiantes - o a razas o grupos étnicos o clases sociales, entre otros- como seres intrínsecamente infantiles, frágiles, regresivos, débiles, ingenuos e impotentes, es una forma de moralismo autoritario que pretende presentarse a sí mis$\mathrm{ma}, \mathrm{y}$ ante aquellos a quienes involucra, como caridad y sensibilidad moral. Se basa en la premisa de que se debe proteger a esos otros. Desafortunadamente, desde esta perspectiva, los "otros" son "cuidados" ya sea que quieran o no serlo -porque después de todo, desde un primer momento los juicios de aquellos a quienes se protege son asumidos como irrelevantes. En el mejor de los casos, esto simplemente perpetúa sentimientos personales de impotencia e inadecuación; en el peor de los casos, y más comúnmente, lesiona cualquier sentido de poder o legitimidad que los individuos puedan haber tenido. Este moralismo autoritario divide en dos cualquier tipo de sociedad libre y excluye cualquier posibilidad de igualdad en una comunidad. Constituye una de las formas más extremas de elitismo ya que se basa en supuestos de diferencias intrínsecas y permanentes, y porque permea todos los dominios de la vida -después de todo, los niños deben ser protegidos por su propio bien en todas las áreas de su vida.

El paternalismo favorece directamente muchos excesos subsidiarios. Primero, anula explicitamente la distinción entre ética y voluntad: cuidar a infantes es protegerlos, aún en contra de su propia voluntad, para prevenir violaciones éticas. En el aspecto que estamos discutiendo, se da una violación flagrante del derecho fundamental a la intimidad que cualquier adulto tiene. Entre los derechos de los ciudadanos adultos a la igualdad en sociedad, está el derecho a tomar las propias decisiones aun cuando los protectores autodesignados arguyan que se trata de decisiones equivocadas. Sólo consideraciones serias, cuidadosas y racionalmente constituídas $-y$ abiertamente debatidas- pueden justificar violaciones de este derecho. Tal debate abierto, por supuesto, no es patrocinado por principios éticos ofuscados y vacios puestos al servicio de valo-res paternalistas encubiertos.

Un segundo exceso moralista se deriva de la instancia paternalista y consiste en la abolición de cualquier distinción entre cuestiones éticas y de voluntad, por un lado, y cuestiones de intrusión legitima de interés por parte de una autoridad constituída -o no constituidapor otro. Ni la inmoralidad ni la falta de voluntad justifican per se la intrusión del estado, o de un "comité de examen" de psicólogos, o de la comunidad de psicólogos, en la vida privada de los adultos. Ciertamente las intrusiones son justificadas, pero no en virtud de la inmoralidad o la falta de juicio únicamente, pues se requieren muchas más condiciones para ello. El paternalismo, por supuesto, presupone la legitimidad de tal intrusión en cualquiera y todos los casos en los que el paternalista siente que hay inmoralidad o falta de juicio involucrados. El paternalismo intrínsecamente excluye el derecho a la privacidad y a la autodeterminación en la "búsqueda de la felicidad" de aquel a quien se protege- al igual que se excluyen dichos derechos a los niños. Tanto en la naturaleza del paternalismo como en principios éticos vacios, se excluyen, inherentemente, consideraciones sobre el derecho a la interferencia -o la falta de ese derecho- en la vida de los demás. Simplemente, nunca se considera. El paternalismo destruye la relevancia de las libertades individuales.

Más aún, en una atmósfera paternalista nunca hay condiciones para discusiones abiertas y racionales en las que -de acuerdo con un curso normal de los eventos- las opresiones y los problemas del paternalismo puedan ser cuestionados. Una discusión abierta y 
racional, precisamente, requiere de respeto y legitimización de todos aquellos involucrados to cual es desde el principio excluido, por la autoafirmación de poder y legitimidad que el paternalista se acredita. La perpetuación de actitudes y posturas paternalistas depende de hecho de evitar o suprimir la pregunta "¿quién vigilará a los vigilantes?". El patemalismo anula toda discusión en la que surjan tales preguntas, negando la igualdad sobre la que dichas discusiones deben basarse.

Un caso paradigmático de tal confusión paternalista es el de las relaciones de pareja establecidas entre profesores y alumnos. Ciertamente, esto involucra un diferencial de poder por ser una relación dual con riesgos inminentes -incluyendo riesgos de explotación antietica o de intentos de explotación. Incluso, es algo que puede ser considerado improcedente. Pero ninguna de estas consideraciones -per sejustifica cualquier intrusión en la vida de los individuos involucrados. Sólo una presunción paternalista puede llevar a la conclusión de que tal intrusión es justificada $y$, en tal caso, con frecuencia se desconoce que los derechos de los estudiantes están siendo violados, que los estudiantes están siendo tratados como niños incompetentes a quienes se puede controlar y en cuyas vidas es posible involucrarse con el argumento de que se les está cuidando por su propio bien.

Otro paradigma de relevancia es el de las relaciones terapéuticas entre profesores y alumnos. De nuevo, hay que tener en cuenta que hay riesgos potenciales, pero también, que es una cuestión que no concierne a nadie más que a los que están directamente involucrados. Es aún más perjudicial la intrusión paternalista en la decisión del estudiante de si debe o no comenzar una relación terapéutica con un miembro de su profesorado, que los riesgos implícitos que tal relación pudiera tener. La actitud paternalista viola directamente la legitimidad y la integridad, e ignora la madurez de las personas objeto del paternalismo - to cual es un perjuicio explicito para cualquier involucrado.

La educación que se proporcione acerca de los riesgos posibles, los derechos de los demás -por ejemplo, guias y criterios de cautela- es el enfoque apropiado que se debe dar a estas cuestiones relativas a la autonomia de los individuos. El paternalismo, por el contrario, inhibe y viola desde el principio tal autonomía.

Es un hecho, sin embargo, que existen muchos que quieren ser cuidados -que buscan el sentido de pertenencia, seguridad y certidumbre de ser protegidos, y que, por tanto están listos a sacrificar sus propios derechos al igual que los derechos de los otros, al servicio de tal beneficio de refugio y protección. Se trata de aquellos que, en otras palabras, intentan permanecer niños y evadir las responsabilidades adultas. $Y$, aunque esta postura presume evidentemente un nivel inferior al del ser humano adulto, el costo y el insulto de ser objeto de paternalismo no se sienten con la misma fuerza. Es un autoengaño existencial en que se incurre para evitar tomar las propias decisiones y cometer los propios errores, que resulta bastante dramático. Pero sin importar qué tan dramático, ni qué tan fuerte y qué tanto sentido tenga ese deseo de evadir la responsabilidad adulta, no puede erigirse como un juicio ético para acogerse o defenderse de otras personas. En ningún terreno es válido como criterio para imponer como obligación ética que haya algunos que deseen someterse al paternalismo.

La noción de relación dual, por supuesto, está diseñada idealmente al servicio de tales actitudes y valores paternalistas. Son actitudes que, de hecho, parecen haber sido útiles en la constitución inicial del principio vacio del que hablamos: la noción de relación dual da la apariencia de una preocupación por el miembro más débil en una relación en la que hay un diferencial de poder involucrado. Pero debido al hecho de que se trata de un principio vacio, es una noción que da vía libre al paternalismo encubierto -o cualquier otro valor conveniente que sea enmarcable dentro del marco de la relación dual.

Otra palabra muy sonada, invocada al servicio de tales valores, es la de "profesionalismo". Como un principio moral o ético per se, profesionalismo es una palabra ambigua -consideremos la noción de profesionalismo aplicada a los guardias de los campos de concentración. Claramente, la palabra puede ser invocada al servicio de otros valores, los cuales deben ser explicitos, defendibles y abiertos a debate por derecho propio. Pero argüir 
"profesionalismo" no es una forma legitima de cerrar el debate.

Se ha insinuado que a mayor poder $y$ conocimiento en un profesional a cargo de una relación con un cliente, hay mayor responsabilidad de su parte que de parte del cliente en el sostenimiento de tal relación. Esto es probablemente correcto y digno de ser enfatizado. Pero también se dice que una relación profesor-alumno es una relación profesional-cliente al igual que lo es la de terapeuta-paciente. $Y$ esta noción es potencialmente atractiva, pero ciertamente más debatible, sin ser establecida de manera a priori. En particular, depende profundamente de caracterizaciones más especificas de lo que es "profesionalismo" -y de la resolución de la ambigüedad del concepto de profesionalismo.

Desafortunadamente, una perspectiva muy común de la relación profesional-cliente se construye sobre la base de que el profesional ejerce un conocimiento superior y está más habilitado que su cliente para decidir sobre "el propio bien" de este último. Esto constituye una construcción trágica para casi todas las instancias que involucran profesionalismo $-y$, ciertamente, es igualmente trágica en la relación terapeuta-cliente y en la de profesor-alumno-. Constituye una postura fundamentalmente patemalista hacia el cliente o el estudiante. El "cliente", según esta perspectiva, ha perdido su derecho a tomar sus propias decisiones y ha sido infantilizado. En terapia, esto es algo que perjudica el autorrespeto y la legitimidad del cliente; en la educación, atenta contra el autorrespeto y la legitimidad del estudiante.

En la educación, esta visión de profesionalismo operando al servicio del cliente también reduce las funciones de los miembros del profesorado a una labor meramente instrumental y limitada -lo que es una visión bastante pobre de la educación-. Se pierde la riqueza y la fascinación del cuestionamiento, el reto, el descubrimiento, la critica, la falseabilidad, el mejoramiento y todos los otros aspectos vitales de la educación genuina. Se pierden todos los valores esenciales del conocimiento y de la búsqueda de éste. En la medida en que lo "profesional" es tomado como algo que implica algo que se hace sobre el cliente, más que con el cliente, el aplicarlo a la educación es algo insultante. Si, por ejem- plo, lo "profesional" acarrea connotaciones de guia a lo largo de un excitante y arriesgado camino en donde la exploración se hace conjuntamente, entonces las inquietudes mencionadas no surgen y -en la misma medida- el paternalismo no se ejerce.

Se ha sostenido, sin embargo, que la persona responsable en la relación (profesional) profesor-estudiante es el profesor. $Y$ esto es bastante escandaloso. Tal responsabilidad seria apenas defendible como una postura hacia los niños - los niños también pueden ser objeto de paternalismo, de condescendencia y también de deshumanización, lo que de hecho, se encuentra como uno de los daños más comunes proferidos en la infancia-; pero pretender que esta postura de responsabilidad sea asumida con los estudiantes es paternalismo en su forma más peligrosa. Es algo que nominalmente se hace por el bien del estudiante, pero que de hecho lo despoja de sus derechos a la privacidad, a tomar decisiones libres y a someterse al poder legitimo que hay en una relación profesor-estudiante. Es un manejo directo y equivoco del poder sobre el estudiante, bajo la apariencia de algo que se hace 'por su bien'. Un estudiante, como cualquier otra persona, debe estar alerta ante cualquiera que venga a hacerle el bien. Thoreau sugiere que la respuesta apropiada en un caso asi seria la de "correr para salvar la propia vida".

Una manifestación contemporánea de la actitud paternalista, generalizada a nivel institucional, es la propuesta de juzgar como oficialmente antiética, cualquier relación sexual entre un terapeuta y cualquier persona que alguna vez haya sido su cliente en cualquier momento del pasado. Aqui el supuesto del paternalismo y la noción de relación dual van de la mano: prohibir una relación entre un terapeuta y alguien que anteriormente fue su cliente, únicamente sobre la base del principio de relación dual, implica correr el riesgo de exponer cualquier otro tipo de relación a la misma vacuidad peligrosa que la aplicación de dicho principio supone. Lo que hace falta es una instancia en la que el concepto de relación dual pueda ser aplicado en forma parecida al paradigma del paralelo terapiarelación sexual y, por consiguiente, continúe confundiendo con su vacuidad fundamental. En este punto, el paternalismo básico infan- 
tilizante viene al rescate: una vez que se ha sido cliente, se seguirá siendo cliente -por tanto, siempre requerirá ser cuidado por una institución o por benefactores externos- a pesar, e incluso a costa, del conocimiento, el consentimiento, la petición del individuo o cualquier otro aspecto incompatible con la, por definición, persona incompetente que se supone que es. Esta persona tan patética, con razón necesitará ser cuidada por benefactores externos y protegida contra explotadores potenciales con quienes tenga relaciones de poder diferenciales. Más aún, cualquier persona así de infantil, necesariamente estará en una seria diferencia de poder en la relación con su exterapeuta -por esto la supuesta necesidad de ser protegida y asi, la aplicabilidad del principio de relación dual-. Es decir que, la infantilización del cliente, que es inherente a una actitud paternalista general, sirve como terreno para demostrar la supuesta aplicabilidad del principio de relación dual al servicio de tal paternalismo. Los niños, después de todo, son el paradigma de las personas que necesitan ser cuidadas -por su propio bien e incluso si se requiere, a costa de sus propios deseos-. Si alguno de los supuestos de esta desagradable cadena de razonamientos y motivaciones fuera cierto -si la terapia siempre sometiera inherentemente a los pacientes a ser menos adultos- entonces la terapia deberia ser prohibida por constituir un azote para la humanidad.

Se ha sugerido que un motivo para la propuesta de "una vez que se es cliente, se es cliente por siempre" es el asegurar que los terapeutas no terminen una relación terapéutica en favor de una eventual relación sexual con sus clientes. Esto es, según esta sugerencia, que el intento no debe ser mantener o presumir que los ex-clientes son intrínsecamente infantiles y que permanentemente necesitan ser cuidados, sino más bien, protegerlos de influencias explotadoras indebidas por parte de los terapeutas en el contexto de una relación terapéutica -una influencia indebida por parte del terapeuta durante la terapia podria producir una explotación antiética de la relación en favor de una relación sexual que técnicamente se comenzaría después de terminada la relación terapéutica-. En otras palabras, puede haber un tecnicismo invo-lucra- do en la prohibición de terapia-relación sexual en donde pudieran propiciarse relaciones de explotación, con los terapeutas salvándose de ser caracterizados y sancionados por ello, sólo por el hecho de que la relación terapéutica estuviera técnicamente terminada. La expresión "una vez que se es cliente, se es cliente por siempre" ciertamente da lugar al sancionamiento oficial de explotaciones de ese estilo. La insinuación de esta afirmación, por supuesto, es la de que el principio de "una vez cliente, se es cliente por siempre", no sería tomado en serio $y$, por tanto, no seria aplicado en situaciones en donde no pudiera hablarse de explotación de la relación terapéutica original. En otras palabras, la premisa se adoptaria como tal, como una forma general de otorgar poderes rápidos a quienes censuran bajo el supuesto de que los usarian únicamente para causas justas.

Una simple prohibición ética hecha oficialmente por la APA en contra de un respiro por parte del terapeuta, lograria el mismo objetivo sin tanto ofuscamiento. Podriamos confiar a los comités de ética la aplicación de este principio solamente en casos real y claramente antiéticos -fueran los que fueran- y todos "dormiriamos tranquilos" sabiendo que tales problemas se evitarían. La racionalidad para el principio "una vez cliente, siempre cliente", es absurda en el más peligroso aunque ingenuo de los extremos. Oscurece totalmente cualesquiera que sean los principios de aplicabilidad que se intenten, pues por ser un principio arbitrario y vacio, puede distorsionarse ampliamente con el tiempo, con interpretaciones y reinterpretaciones, políticas, gustos, disgustos y caprichos personales y todas las inevitables influencias de ese estilo. La vacuidad del principio de relación dual se recrea aqui simple $y$, al menos parcialmente, con base en el supuesto de que no todas las instancias del principio deben ser 0 , de hecho, serán sancionadas.

La fe ingenua en que tan arbitrario poder será siempre ejercido "por el bien" de otros es aterrador. No lo es, de hecho, si usted se asume a si mismo como parte de aquellos que aplican los principios por el bien de unos $u$ otros, pero si si se coloca en el paquete de los que están siendo protegidos o sancionados por violar el principio. Es decir, no es aterrador 
mientras usted se asuma como parte de la élite de los verdaderos adultos, que tienen fervor y afición moral, santurroneria y humildad propia y sentimiento del deber de cuidar a todos aquellos patéticos y pobres indefensos infantes -los ex-clientes- del mundo.

Estoy usando un fuerte sarcasmo en estas palabras. Algunos con seguridad sentirán que estoy siendo desmesuradamente fuerte --que, incluso si el análisis es correcto, las posiciones criticadas son tomadas de buena fe $y$, en el peor de los casos, son errores bien intencionados por parte de los involucrados. Este argumento se fundamenta en el supuesto de que las buenas intenciones presumiblemente involucradas dejan los crimenes cometidos, si los hay, moralmente no viables. Como respuesta, yo cito la renombrada banalidad de la maldad. Mucha maldad, de hecho, se basa en serias buenas intenciones mal guiadas, y son perpetradas y ejecutadas por funcionarios banales. La Inquisición y Adolf Eichmannn son ejemplos pragmáticos de "buenas intenciones" y banalidad. Mi argumento es, precisamente, que la total vacuidad de principios tales como el de las relaciones duales, y el rampante paternalismo oculto que implica, han estimulado y perpetrado daños y perjuicios -daños y perjuicios moralmente reprensibles-. Estas actitudes y orientaciones en el terreno son fuente peligrosa de maldad oficial y semi-oficial.

En el más abstracto, pero quizás más importante de los niveles, los principios vacios que meramente sirven para ocultar las bases reales de una decisión, no pueden ser útiles sino inhibidores de una exploración y debate racional de la ética involucrada -en cualquier relación en cuestión. Esto es una profunda y seria supresión de los derechos de la sociedad civil, y un entrenamiento profundamente peligroso para los estudiantes en cuanto a que acepten irracionalmente los edictos y la autoridad. Es un entrenamiento para el vivir -al menos para el vivir profesional- en términos de dogma, no en términos de deliberación racional y critica. Un dogma, sin embargo, es siempre perjudicial, y su promulgación es intrínsecamente antiética.

Aún más directamente, los casos paradigmáticos sobre los que se han construido éstos principios -relaciones explotadoras entre terapeutas y clientes- envuelven fuertes $y$ justificadas emociones. Sin embargo, sin la clarificación de un discurso abierto, crítico y racional, la intensidad de dichas emociones es aplicada, además, sobre cualquiera que cuestione o tenga dudas acerca de dichos principios vacios. El resultado aqui no es simplemente una inhibición de la discusión racional en virtud del hecho de que los principios reales y los valores involucrados están ocultos, sino la supresión explicita de la discusión por intimidación emocional o culpabilizante --la intimidación por la intensidad de la reacción emocional ante una critica o duda sobre dichos principios vacios es sentida como una defensa de o excusa para la explotación sexual. Es la intimidación por medio de la culpa de quienes critican o dudan de dichos principios acusándoles de estar defendiendo o excusando algo en beneficio de los derechos de los intrinsecamente machos rapaces. Esta intimidación impone serias violaciones básicas de respetuo mutuo y discusión abierta. Esta intensidad, de hecho, puede ser mitigada mediante proteccionismo hacia el individuo que está cuestionando o dudando -él o ella es ignorante o simplemente está equivocado y por ello necesita ser guiado hacia el camino correcto del pensamiento y los valores. Este paternalismo es aún más sutil que la condenación directa, porque combina la confusión conceptual de principios vacios con la confusión moral de actitudes paternalistas.

Hay aún otro nivel de evolución en lo perjudicial de este "cáncer". La santurronería que es inherente a la actitud paternalista, junto con la intensidad emocional de las cuestiones éticas paradigmáticas, más la vacuidad conceptual y la supresión de racionalidad inherente en los supuestos principios éticos, y el autoritarismo intrinseco con el que dichas posiciones son presentadas, se combinan para producir una atmósfera social de moralidad excesiva, fanática e irracional. La consecuencia directa es una vigilancia chismosa, maliciosa, odiosa e hiriente entre aquellos atrapados en el dogma prevaleciente. En esto, no solamente hay consideraciones reales escondidas, y no solamente se enseña la condescendencia del paternalismo, sino que se promueve la tragedia ética de mojigatería fanática en las masas. 


\section{UN PRINCIPIO ÉTICO ALTERNATIVO}

No he criticado, ni criticaré, la intuición básica de que una mezcla de terapia y relación sexual es algo intrínsecamente antiético. Desafortunadamente, cuando intuiciones válidas como ésta son fundadas en principios vacios como el de las relaciones duales, la denuncia de tal vacuidad deja la intuición subyacente carente de una verdadera racionalidad. Esto, en si mismo, es peligroso: cualquier reconocimiento de tal vacuidad amenaza el soporte y la fuerza de una conclusión ética.

Yo quiero ofrecer, para consideración, un principio ético alternativo que apoye dicha intuición. Es un principio que es intrínseco a la naturaleza de lo que es una relación terapéutica. Es decir, yo resalto la inherencia de una naturaleza ética que hay en la relación terapéutica per se, y no en un principio ético aplicable a la relación terapéutica. Si esto es válido, entonces este principio es mucho más fuerte y convincente que la aplicación del principio vacio de las relaciones duales.

Consideremos primero la pregunta de si una relación sexual puede o no ser también una relación terapéutica. La respuesta, espero, es "sí". Si no, la vida de matrimonio estaría en serios problemas. Pero esto da lugar a la siguiente pregunta acerca de qué diferencia existe entre relación terapéutica -caracteristica que una relación sexual también puede tener- y una relación de terapia.

En una relación terapéutica, entre otras cosas, se alcanza un propósito o función terapéutica. Una relación de terapia es una relación contractualmente obligada a maximizar la función terapeutica. Es precisamente dicha obligación lo que para mí constituye una relación como relación de terapia, y no como otro tipo de relación.

Dentro de las caracteristicas más importantes de una relación de terapia al servicio de tal obligación están las limitaciones intrinsecas que tiene. Es decir, las limitaciones relativas a las condiciones definidas de compromiso, tiempo y reducción de asuntos personales entre el terapeuta y el cliente, como una forma de proteger la habilidad de ambos, terapeuta y cliente, para explorar las cuestiones del cliente con mínimas intrusiones y distorsiones de aspectos externos no relacionados. Estos asuntos externos nunca pueden ser enteramente eliminados, pero, entre las obligaciones fundamentales del terapeuta, está la de minimizar sus intrusiones.

Contrariamente, es una violación ética y contractual de la relación de terapia permitir -y ciertamente promover o dar lugar a- la intrusión de dichos aspectos externos en el ámbito de la relación de terapia. Esta violación de los limites de la relación de terapia efectivamente la destruye como tal. Destruye la protección involucrada en la obligación de minimizar tales irrupciones, porque los asuntos externos se involucran. La protección sólo funciona como una ayuda, un soporte para la confianza y seguridad del cliente -y del terapeuta- de que tales asuntos extemos serán minimizados y por tanto, no tendrán que ser tomados en cuenta. La confianza y la seguridad son irremediablemente destruidas, es decir, su obligación es irremediablemente violada, con la intrusión de asuntos sexuales.

Esta obligación de minimizar asuntos externos ajenos a la terapia, es independiente de cualquier forma, explotadora o no, en que dichas cuestiones puedan involucrarse. Un terapeuta, como terapeuta, tiene la obligación intrinseca de minimizar cualquier intrusión, sin importar la manera en que se presenten. Una intrusión ejercida en forma explotadora constituye una violación ética adicional a la intrusión en si misma.

La explotación, en cualquier relación -terapia-cliente, profesor-alumno o cualquier otraes en si misma e intrínsicamente antiética. Nada en este análisis debe ser tomado como que se está descontando o minimizando la importancia o validez de tales consideraciones y principios éticos adicionales. Lo que yo estoy proponiendo, además del rango amplio de consideraciones éticas que puede ser aplicado a la relación de terapia, es un principio ético que es inherente y especifico de la relación de terapia per se (Bickhard, 1989).

Notemos que este principio conlleva un sentido intrínseco por el cual el paralelo terapia-relación sexual es antiético -con la posible excepción de las relaciones sexuales subrrogadas, en las cuales la sexualidad nace parte del contrato desde el principio. La voluntad o eficacia de tales relaciones sexuales subrrogadas - 0 su carencia- no es abordada por tales consideraciones. 
Es importante enfatizar que la obligación es minimizar los asuntos externos, mas no asegurar su ausencia. Estos no pueden ser evitados del todo, y algunas relaciones los incluirán más que otras. Ejemplos incluyen las obligaciones éticas y legales de advertir a otros sobre peligros potenciales, o las relaciones de terapia entre individuos que ya tienen una o más relaciones. La obligación ética involucrada es la de minimizar, no eliminar, la intrusión de cuestiones extemas. Esto incluye una obligación de informar y educar a los individuos en lo concerniente a los riesgos potenciales involucrados en tales relaciones. Esto, para mi, es la perspectiva ética correcta en caso de relaciones de terapia entre individuos que ya se conocen -como en sectores de provincia o comunidades pequeñas- 0 entre profesores y estudiantes. No es propio -de hecho, es paternalista y dañino- prohibir tales relaciones de terapia.

El principio que yo ofrezco, entonces, no apoya todas las instancias que uno desearia cubrir bajo el principio de relaciones duales. Sugiero, sin embargo, que aquellas instancias excluidas no son necesariamente antiéticas. Claramente pueden ser antiéticas -cualquier relación puede serlo-, pero el principio de relación dual ha sido usado como si fuera algo inherente e inevitablemente antiético, y eso es inaceptable. No se puede juzgar válidamente como antiético algo cuya base está en un principio vacio de relación dual, o sobre cualquier otro de sus valores paternalistas de base. Como minimo, cualquiera de aquellos argumentos de violaciones éticas requiere un reexamen cuidadoso de sus bases a la luz de las consideraciones presentadas aquí.

\section{CONCLUSIÓN}

La intuición central y paradigmática del paralelo terapia-relación sexual como algo an- tiético es, para mi, correcta. Desafortunadamente, se la ha basado en principios y valores que son vacios, confusos, paternalistas y dañinos. El paternalismo, en particular, es deshumanizante así se trate de clientes, ex-clientes, estudiantes u otros -incluidos niños. Este complejo de actitudes, valores y falta de logica debe ser confrontado. Aún más, desafortunadamente uno de los daños causados por este complejo es precisamente la inhibición, la represión y el castigo infringidos a quienes se atreven a cuestionarlos -la abolición de la razón, la critica y la discusión.

Una vez confrontada, sin embargo, la intuición básica concerniente al paralelo terapiarelación sexual aparentemente queda sin un soporte racional. Yo ofrezco un soporte racional alternativo para esta intuición, que es mucho más fuerte, y mucho más intrínseco a la naturaleza de la terapia per se, que los estándares racionales. Es en efecto, "una simple" explicación de un aspecto de la relación de terapia, que es el de su carácter intrinsecamente ético.

Más importante aún es mi deseo de estimular la discusión crítica y racional de tales cuestiones y la creación de una atmósfera facilitadora de tal discusión. De otra manera, la consecuencia más insidiosa de las cuestiones "éticas" dominantes en este campo es un autoritarismo paternalista y la resultante supresión -algunas veces, viciosa- del debate racional de tales cuestiones en si mismas.

\section{REFERENCIAS BIBLIOGRÁFICAS}

Bickhard, M.H. (1989). Ethical psychotherapy and psychotherapy as ethics: A response to Perrez. New ldeas in Psychology, 7(2), 159-164.

\section{NOTAS}

'Traducción de Sonia Jiménez Suárez 